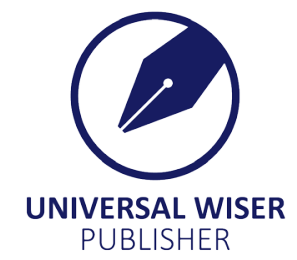

\title{
The Performance of the EWMA Median Chart in the Presence of Measurement Error
}

\author{
Huu Du Nguyen ${ }^{1}$, Quoc Thong Nguyen ${ }^{2 *}$, Thi Hien Nguyen $^{3}$, Narayanaswamy Balakrishnan ${ }^{4}$, Kim Phuc \\ $\operatorname{Tran}^{5}$ \\ ${ }^{1}$ Department of Mathematics, Vietnam National University of Agriculture, Hanoi, Vietnam \\ ${ }^{2}$ Institute of Artificial Intelligence and Data Science, Dong A University, Da Nang, Vietnam \\ ${ }^{3}$ Departement of Mathematics, CY TECH, CY Cergy Paris University, 95000 Cergy, France \\ ${ }^{4}$ Department of Mathematics and Statistics, McMaster University, Hamilton, ON L8S 4K1, Canada \\ ${ }^{5}$ Ecole Nationale Sup'erieure des Arts et Industries Textiles, GEMTEX Laboratory, France \\ Email: thongnq@donga.edu.vn
}

\begin{abstract}
Measurement error always exists in quality control applications and may considerably affect the ability of control charts to detect an out-of-control situation. In this paper, we study the performance of the EWMA median chart using a Markov Chain approach with a linear covariate error model and a corrected formula for the distribution of the sample median. The performance is evaluated through the Average Run Length. Several figures and tables are presented and enumerated to show the statistical performance of the EWMA median control chart for different sources of measurement errors. The positive effect of taking multiple measurements for each item in a subgroup on the performance of the EWMA median chart is also investigated. An example illustrates the use of this study is introduced. The numerical simulation shows that the measurement errors have a negative influence on the proposed chart.
\end{abstract}

Keywords: measurement error, median, EWMA control chart, expert systems, smart production

\section{Introduction}

In manufacturing industries, a process of product can be affected by many assignable causes. Detecting the anomalies as soon as possible is one of the producer's top priorities to ensure the product quality. Therefore, many anomaly detection techniques have been developed ${ }^{[30,28]}$. One of the most widely used techniques is control charts. Control charts are the simplest type of on-line statistical process control (SPC) procedure used to detect variations in parameters of processes. In the SPC literature, the median $(\tilde{X})$ type charts have already been investigated and used to detect shifts in a process. As emphasized in [6], the main advantages of median type charts are that they are simpler than the corresponding mean $(\tilde{X})$ charts and they are robust against outliers, contamination or small deviations from normality. Castagliola ${ }^{[4]}$ was the first to introduce the EWMA median chart while the Shewhart- $\tilde{X}$ control chart was suggested in [16]. Both of these control charts are robust and effective tools for practitioners who are only interested in the detection of permanent shifts in the process mean. As an effort to improve the statistical performance of median type control charts, the generally weighted moving average median (GWMA- $\tilde{X}$ ) control chart has been studied in [23]. In practice, the parameters of control charts are usually unknown and estimated from an in-control sample. Castagliola and Figueiredo ${ }^{[5]}$ proposed a modified Shewhart median chart with estimated control limits and showed that the in-control performance of the median charts with estimated control limits is different when the process parameters are assumed known. Very recently, Castagliola et al. ${ }^{[6]}$ developed a new EWMA- $\tilde{X}$ chart with estimated control limits to monitor the mean value of a normal process.

In many industrial scenarios, the presence of measurement error can seriously affect the performance of control charts. The performance of various control charts in the presence of measurement error has been shown by the works of several authors, including ${ }^{[1,1,13,20,15,14,26,27,22]}$. For the case of the EWMA- $\tilde{X}$ control chart, the efect of measurement error on its performance has been considered in [10]. However, in that paper, the authors applied a mistaken formula for the cumulative density function of the sample median in the presence of measurement error. Therefore, the goal of this paper is to correct this formula and to investigate the true efect of measurement error on the EWMA- $\tilde{X}$ control chart's performance. This correction is needed to avoid confusion among readers.

Copyright (C2020 Huu Du Nguyen, et al.

DOI: https://doi.org/10.37256/aie.112020401

This is an open-access article distributed under a CC BY license

(Creative Commons Attribution 4.0 International License)

https://creativecommons.org/licenses/by/4.0/ 
The outline of the paper is as follows. In section 2, the linear covariate error model for the sample median is defined, along with its corrected distribution. Section 3 presents the implementation of the EWMA- $\tilde{X}$ with measurement error where the run length performances $A R L$ and $S D R L$ are defined by using a Markov Chain-based approach. In Section 4, the effect of measurement error on the EWMA- $\tilde{X}$ control chart performance is investigated. Section 5 presents an illustrative example and, finally, concluding remarks and recommendations are given in Section 6.

\section{Linear covariate error model and sample median distribution}

\subsection{Linear covariate error model}

Using the general linear covariate error model suggested in Linna and Woodall ${ }^{[18]}$, Tran et al. Kim Phuc Tran et al. ${ }^{[25]}$ provided the linear covariate error model for the sample media $\tilde{X}$. From the observable quantities $\left\{X_{i, j, 1}^{*}, X_{i, j, 1}^{*}, \ldots, X_{i, j, m}^{*}\right\}$, where $X_{i, j, k}^{*}$ represents the $k^{t h}$ measurement of the $j^{t h}$ sample at time $i, k=1, \ldots, m, j=1, \ldots, n$, $i=1,2, \ldots$, the mean $\tilde{X}_{i, j}^{*}$ is determined, which is a normal distribution. In particular, $\tilde{\boldsymbol{X}}_{i, j}^{*} \sim N\left(\mu^{*}, \sigma^{*}\right)$ with

$$
\begin{aligned}
& \mu^{*}=A+B\left(\mu_{0}+\delta \sigma_{0}\right), \\
& \sigma^{*}=\sqrt{B^{2} \sigma_{0}^{2}+\frac{\sigma_{M}^{2}}{m}},
\end{aligned}
$$

where $A$ and $B$ are the two coefficients in the linear covariate error model; $\sigma_{\mathrm{M}}$ is the standard deviation of the random error term in the model, $\mu_{0}$ and $\sigma_{0}$ are the nominal mean and standard-deviation; and $\delta$ is the mean shift. Let $\tilde{X}_{i}^{*}$ be the sample median of the mean values $\left\{\bar{X}_{i, 1}^{*}, \bar{X}_{i, 2}^{*}, \ldots, \bar{X}_{i, n}^{*}\right\}$ corresponding to subgroup $i=1,2, \ldots$ Then, $\tilde{X}_{i}^{*}$ is defined by

$$
\tilde{\boldsymbol{X}}_{i}^{*}\left\{\begin{array}{lc}
\overline{\boldsymbol{X}}_{i,((n+1) / 2)}^{*} & \text { if } n \text { is odd, } \\
\frac{\overline{\boldsymbol{X}}_{i,(n / 2)}^{*}+\overline{\boldsymbol{X}}_{i,(n / 2+1)}^{*}}{2} & \text { if } n \text { is even, }
\end{array}\right.
$$

where $\left\{\bar{X}_{i,(1)}^{*}, \bar{X}_{i,(2)}^{*}, \ldots, \bar{X}_{i,(n)}^{*}\right\}$ is the ordered sample of the mean values forsubgroup $i=1,2, \ldots$

\subsection{Sample median distribution}

The sample median distribution has been provided and applied broadly in the literature. In Cheng and Wang ${ }^{[10]}$, the authors applied asymptotic formula for the variance of sample median when $n$ is odd, i.e.

$$
V\left(\tilde{X}_{i}^{*}\right)=\frac{\pi}{2 n}\left(B^{2} \sigma_{0}^{2}+\frac{\sigma_{M}^{2}}{m}\right) .
$$

As a result, when the sample size $n$ is odd, the cumulative distribution function $(c d f)$ of the sample median $\tilde{X}_{i}^{*}$ is reported as (see Eq. (5) in Cheng and Wang ${ }^{[10]}$ )

$$
\begin{aligned}
F_{\tilde{X}_{i}^{*}}(x, n=o d d) & =F_{\text {beta }}\left[\Phi\left(\frac{x-\mu^{*}}{\sqrt{\pi / 2 n \sigma^{*}}}\right) ; \frac{n+1}{2}, \frac{n+1}{2}\right] \\
& =F_{\text {beta }}\left[\Phi\left(\frac{x-A-B\left(\mu_{0}+\delta \sigma_{0}\right)}{\sqrt{\frac{\pi}{2 n}\left(B^{2} \sigma_{0}^{2}+\frac{\sigma_{M}^{2}}{m}\right)}}\right) ; \frac{n+1}{2}, \frac{n+1}{2}\right],
\end{aligned}
$$


where $\Phi($.$) is the c d f$ of the standard normal distribution and $F_{\text {beta }}(x ; a, b)$ is the $c d f$ of beta distribution with parameters $\left(\frac{n+1}{2}, \frac{n+1}{2}\right)$. Similarly, when the sample size $\mathrm{n}$ is even, the cdf of the sample median is (see Eq. (6) in Cheng and Wang ${ }^{[10]}$ )

$$
\begin{aligned}
& F_{\tilde{X}_{i}^{*}}(x, n=\text { even })=F_{\text {beta }}\left[\Phi\left(\frac{x-\mu^{*}}{\sqrt{\pi / 2 n \sigma^{*}}}\right) ; \frac{n}{2}, \frac{n}{2}+1\right] \\
& -\frac{n !}{(n / 2-1) !(n / 2) !} \int_{-\infty}^{x} F\left(x_{2 / n}\right)^{n / 2-1}\left[1-F\left(2 x-x_{2 / n}\right)\right]^{2 / n} f\left(x_{2 / n}\right) d x_{2 / n}
\end{aligned}
$$

where $F\left(x_{2 / n}\right)$ and $f\left(x_{2 / n}\right)$ are the cdf and the probability density function ( $\left.p d f\right)$ of the order statistic $\bar{X}_{i, n / 2}^{*}$.

However, it should be considered that the formula (4) is an asymptotic one. It can only be used to approximate the distribution of the sample median when the sample size is sufficiently large, which is not the case in practice when the sample size only takes not so large value such as $n=3, n=5$ or $n=7$. Moreover, when using the asymptotic distribution of the sample median, it is not a beta distribution with the added incorrect term $\frac{\pi}{2 n}$ as given in Cheng and Wang ${ }^{[10]}$. Instead, it is a normal distribution.

Based on the exact probability density distribution ( $p d f$ ) of the sample median when $n$ is odd provided by Chu ${ }^{[12]}$, we have checked that the correct $c d f$ of sample median is

$$
\begin{aligned}
F_{\tilde{X}_{i}^{*}}(x, n \quad \text { odd }) & =F_{\text {beta }}\left[\Phi\left(\frac{x-\mu^{*}}{\sigma^{*}}\right) ; \frac{n+1}{2}, \frac{n+1}{2}\right] \\
& =F_{\text {beta }}\left[\Phi\left(\frac{x-A-B\left(\mu_{0}+\delta \sigma_{0}\right)}{\sqrt{B^{2} \sigma_{0}^{2}+\frac{\sigma_{M}^{2}}{m}}}\right) ; \frac{n+1}{2}, \frac{n+1}{2}\right],
\end{aligned}
$$

where $\Phi($.$) is the c d f$ of the standard normal distribution and $F_{\text {beta }}(x ; a, b)$ is the $c d f$ of beta distribution with parameters $(a, b)$. This formula has also been used by other authors ${ }^{[7,25]}$. Similarly, when $n$ is even, the correct distribution of sample median must be (see equation 2.5.10 in [2], for example)

$$
\begin{aligned}
& F_{\tilde{X}_{i}^{*}}(x, n=\text { even })=F_{\text {beta }}\left[\Phi\left(\frac{x-\mu^{*}}{\sigma^{*}}\right) ; \frac{n}{2}, \frac{n}{2}+1\right] \\
& -\frac{n !}{(n / 2-1) !(n / 2) !} \int_{-\infty}^{x} F(t)^{n / 2-1}[1-F(2 x-t)]^{n / 2} f(t) d t .
\end{aligned}
$$

When working with the sample median, it is usual assumed, without loss of generality, that the sample size $n$ is an odd value. This makes the sample median easier and faster to compute. In the rest of this paper, we will thus confine to the case where $n$ is an odd value. That is to say, the formula (7) will be used as the $c d f$ of the sample median.

\section{Implementation of the EWMA- $\tilde{X}$ control chart with measurement error}

In this section, we present the implementation of the EWMA- $\tilde{X}$ control chart with measurement error. Let $Z_{1}^{*}, Z_{2}^{*}, \ldots$ be the EWMA sequence obtained from $\tilde{X}_{1}^{*}, \tilde{X}_{2}^{*}, \ldots$, i.e. for $i \in\{1,2, \ldots\}$,

$$
Z_{i}^{*}=(1-\lambda) Z_{i-1}^{*}+\lambda \tilde{X}_{i}^{*}
$$

where $Z_{0}^{*}=\mu_{0}^{*}$ and $\lambda \in(0,-1]$ is a smoothing constant. The values in the EWMA sequence will be monitored to define whether the process is still in-control. This is performed by comparing these values with the control limits of the EWMA- 
$\tilde{X}$ chart. Similar to Tran et al. ${ }^{[25]}$, the control limits of the EWMA - $\tilde{X}$ chart with measurement error are defined by

$$
\begin{aligned}
& L C L=A+B \mu_{0}-K \sqrt{B^{2} \sigma_{0}^{2}+\frac{\sigma_{M}^{2}}{m}}, \\
& L C L=A+B \mu_{0}+K \sqrt{B^{2} \sigma_{0}^{2}+\frac{\sigma_{M}^{2}}{m}},
\end{aligned}
$$

where $K>0$ is a constant that depends on $n, \lambda$ and on the desired in-control performance.

The run length properties of the EWMA- $\tilde{X}$ chart with linear covariate error model can be obtained using the classical approach proposed by Brook and Evans ${ }^{[3]}$ which consists in dividing the interval $[L C L, U C L]$ into $2 p+1$ subintervals $\left(H_{j}-\Delta, H_{j}+\Delta\right], j \in\{-p, \ldots, 0, \ldots,+p\}$, centered at $H_{j}=\frac{U C L+L C L}{2}+2 j \Delta$ where $\Delta=\frac{U C L-L C L}{2(2 p+1)}$. Using (10) for $L C L$ and $U C L$
leads to

$$
\begin{aligned}
& H_{j}=A+B \mu_{0}+2 j \frac{K \sqrt{B^{2} \sigma_{0}^{2}+\frac{\sigma_{M}^{2}}{m}}}{(2 p+1)}, \\
& \Delta=\frac{K \sqrt{B^{2} \sigma_{0}^{2}+\frac{\sigma_{M}^{2}}{m}}}{(2 p+1)} .
\end{aligned}
$$

Each subinterval $\left(H_{j}-\Delta, H_{j}+\Delta\right], j \in\{-p, \ldots, 0, \ldots,+p\}$, represents a transient state of a Markov chain. If $Z_{i} \in\left(H_{j}-\Delta, H_{j}+\Delta\right]$ then the Markov chain is in the transient state $j \in\{-p, \ldots, 0, \ldots,+p\}$ sample $i$. If $Z_{i}^{*} \notin\left(H_{j}-\Delta, H_{j}+\Delta\right]$ then the Markov chain reached the absorbing state $(-\infty, L C L] \cup[U C L,+\infty)$. We assume that $H_{j}$ is the representative value of state $j \in\{-p, \ldots, 0, \ldots,+p\}$. Let $\mathbf{Q}$ be the $(2 p+1,2 p+1)$ sub-matrix of probabilities $\mathbf{Q}_{j, k}$ corresponding to the $2 p+1$ transient states defined above, i.e.

$$
\mathbf{Q}=\left(\begin{array}{ccccccc}
Q_{-p,-p} & \ldots & Q_{-p,-1} & Q_{-p, 0} & Q_{-p,+1} & \ldots & Q_{-p,+p} \\
\vdots & \vdots & \vdots & \vdots & \vdots & \vdots & \vdots \\
Q_{-1,-p} & \ldots & Q_{-1,-1} & Q_{-1,0} & Q_{-1,+1} & \ldots & Q_{-1,+p} \\
Q_{0,-p} & \ldots & Q_{0,-1} & Q_{0,0} & Q_{0,+1} & \ldots & Q_{0,+p} \\
Q_{+1,-p} & \ldots & Q_{+1,-1} & Q_{+1,0} & Q_{+1,+1} & \ldots & Q_{+1,+p} \\
\vdots & \vdots & \vdots & \vdots & \vdots & \vdots & \vdots \\
Q_{+p,-p} & \ldots & Q_{+p,-1} & Q_{+p, 0} & Q_{+p,+1} & \ldots & Q_{+p,+p}
\end{array}\right) .
$$

By definition, we have $Q_{j, k}=P\left(Z_{i}^{*} \in\left(H_{k}-\Delta, H_{k}+\Delta\right] \mid Z_{i-1}^{*}=H_{j}\right)$ or, equivalently, $Q_{j, k}=P\left(Z_{i}^{*} \leq H_{k}+\Delta \mid Z_{i-1}^{*}=H_{j}\right)$ $P\left(Z_{i}^{*} \leq H_{k}-\Delta \mid Z_{i-1}^{*}=H_{j}\right)$. Replacing $Z_{i}^{*}=(1-\lambda) Z_{i-1}^{*}+\lambda \tilde{X}_{i}^{*}, Z_{i-1}^{*}=H_{j}$ and isolating $\tilde{X}_{i}^{*}$ gives

$$
\begin{aligned}
Q_{j, k} & =P\left(\tilde{X}_{i}^{*} \leq \frac{H_{k}+\Delta-(1-\lambda) H_{j}}{\lambda}\right)-P\left(\tilde{X}_{i}^{*} \leq \frac{H_{k}-\Delta-(1-\lambda) H_{j}}{\lambda}\right), \\
& =F_{\tilde{X}_{i}^{*}}\left(\frac{H_{k}+\Delta-(1-\lambda) H_{j}}{\lambda} \mid n\right)-F_{\tilde{X}_{i}^{*}}\left(\frac{H_{k}-\Delta-(1-\lambda) H_{j}}{\lambda} \mid n\right),
\end{aligned}
$$


From (11), (7) and (12), it can be proven that

$$
Q_{j, k}=F_{\beta}\left(\Phi\left(\frac{(2 k+1-2 j(1-\lambda)) K}{\lambda(2 p+1)}-\frac{B \delta \sigma_{0}}{\sqrt{B^{2} \sigma_{0}^{2}+\frac{\sigma_{M}^{2}}{m}}}\right) \frac{n+1}{2}, \frac{n+1}{2}\right)-F_{\beta}\left(\Phi\left(\frac{(2 k-1-2 j(1-\lambda)) K}{\lambda(2 p+1)}-\frac{B \delta \sigma_{0}}{\sqrt{B^{2} \sigma_{0}^{2}+\frac{\sigma_{M}^{2}}{m}}}\right) \mid \frac{n+1}{2}, \frac{n+1}{2}\right)
$$

Finally, dividing the numerator and the denominator by $\sigma_{0}$ allows to obtain $p$ as a function of the precision error ratio $\eta=\frac{\sigma_{M}}{\sigma_{0}}$

$$
\begin{gathered}
Q_{j, k}=F_{\beta}\left(\Phi\left(\frac{(2 k+1-2 j(1-\lambda)) K}{\lambda(2 p+1)}-\frac{B \delta}{\sqrt{B^{2}+\frac{\eta^{2}}{m}}}\right) \mid \frac{n+1}{2}, \frac{n+1}{2}\right) \\
-F_{\beta}\left(\Phi\left(\frac{(2 k-1-2 j(1-\lambda)) K}{\lambda(2 p+1)}-\frac{B \delta}{\sqrt{B^{2}+\frac{\eta^{2}}{m}}} \mid \frac{n+1}{2}, \frac{n+1}{2}\right)\right.
\end{gathered}
$$

Let $\mathbf{q}=\left(\mathrm{q}_{-p}, \ldots, \mathrm{q}_{0}, \ldots, \mathrm{q}_{p}\right)^{T}$ be the $(2 p+1,1)$ vector of initial probabilities associated with the $2 p+1$ transient states, where

$$
q_{j}\left\{\begin{array}{l}
0 \quad \text { if } Z_{0}^{*} \notin\left(H_{j}-\Delta, H_{j}+\Delta\right] \\
1 \quad \text { if } Z_{0}^{*} \in\left(H_{j}-\Delta, H_{j}+\Delta\right] .
\end{array}\right.
$$

When the number $2 p+1$ of subintervals in matrix $\mathbf{Q}$ is sufficiently large (say $p=100$, i.e. $2 p+1=201$ ), this finite approach provides an effective method that allows the Run Length properties of the EWMA- $\tilde{X}$ chart with linear covariate error model to be accurately evaluated. Since the Run Length of the EWMA- $\tilde{X}$ chart in the presence of measurement error is a Discrete PHase-type (or DPH) random variable of parameters ( $\mathbf{Q}, \mathbf{q})$, see for instance [21] or [17], then the $p d f$ $f_{R L}(\boldsymbol{\ell})$ and the $c d f F_{R L}(\ell)$ of $R L$ are respectively equal to

$$
\begin{aligned}
& f_{R L}(\ell)=q^{T} \mathbf{Q}^{\ell-1} r, \\
& F_{R L}(\ell)=1-q^{T} \mathbf{Q}^{\ell} 1,
\end{aligned}
$$

where $\mathbf{r}=1-\mathbf{Q} 1$ with $\mathbf{1}=(1,1, \ldots, 1)^{T}$ and the mean $(A R L)$, the second non-central moment $E 2 R L=E\left(R L^{2}\right)$ and the standard-deviation $(S D R L)$ of $R L$ are respectively equal to

$$
\begin{aligned}
& A R L=v_{1}, \\
& E 2 R L=v_{1}+v_{2}, \\
& S D R L=\sqrt{E 2 R L-A R L^{2}},
\end{aligned}
$$


where $v_{1}$ and $v_{2}$ are the first and second factorial moments of the $R L$, i.e.

$$
\begin{aligned}
& v_{1}=\mathbf{q}^{T}(\mathbf{I}-\mathbf{Q})^{-1} \mathbf{1}, \\
& v_{2}=2 \mathbf{q}^{T}(\mathbf{I}-\mathbf{Q})^{-2} \mathbf{Q} \mathbf{1} .
\end{aligned}
$$

From (14), it should be considered that the values of $A R L$ and $S D R L$ of the EWMA- $\tilde{X}$ chart with linear covariate error model do not depend on the constant $A$ which implies that this constant does not have any influence on the performance of the EWMA- $\tilde{X}$ control chart with measurement error. Nevertheless, the constant $A$ must be taken into account concerning the process location, the item disposition or the process capability (see Linna et al. ${ }^{[19]}$ ).

\section{The effect of measurement error on the EWMA- $\tilde{X}$ control chart}

In this section, we investigate the effect of measurement error on the performance of EWMA- $\tilde{X}$ control chart represented by the mean and the standard deviation of the run length distribution ( $A R L$ and $S D R L)$. Moreover, the expected average run length $(E A R L)$ is also considered. In Table 1, we presented the published studies on median monitoring with the present of measurement errors.

Table 1. The previous methods on monitoring median with the present of measurement errors

\begin{tabular}{cccc}
\hline Publication & Method & Advantages & Drawbacks \\
\hline Tran et al. $^{[25]}$ & Shewhart & Classical method, easy to implement & Least efficient \\
Cheng and Wang ${ }^{[10]}$ & EWMA and CUSUM & & The formula is not realistic in practice \\
Cheng and Wang ${ }^{[1]]}$ & VSSI & Easier than EWMA & The formula is not realistic in practice \\
Tran et al. $^{[29]}$ & Synthetic & Not difficult to implement & Less efficient than EWMA with small shifts \\
Tang et al. ${ }^{[24]}$ & Adaptive EWMA & Good performance & Complicated to implement \\
\hline
\end{tabular}

As proposed $\mathrm{in}^{[6]}$, the design of the EWMA- $\tilde{X}$ control chart consists in selecting the optimal couples $\left(\lambda^{*}, K^{*}\right)$ which minimize the out-of-control $A R L$ for a specific value of $n$ and $\delta$ subject to a constraint on the in-control $A R L$, which is denoted by $A R L_{0}$ and set at $A R L_{0}=370.4$ in this study. For computational reasons (convergence of the Markov-chain method), the value of $\lambda$ is always kept larger or equal to 0.05 .

Table 2 shows the optimal couples $\left(\lambda^{*}, K^{*}\right)$ of the EWMA- $\tilde{X}$ chart for $B=1$ and $m=1$ and different values of $\eta \in\{0,0.1$, $0.2,0.3,0.5,1.0\}, \delta \in\{0,0.1,0.2,0.3,0.5,0.7,1.0,1.5,2.0\}$ and $n \in\{3,5,7,9\}$.

It is important to note that the optimal couples $\left(\lambda^{*}, K^{*}\right)$ of the EWMA- $\tilde{X}$ control chart for other investigated scenarios are not presented in this paper but are available upon request from authors.

Table 2. $\lambda^{*}$ and $K^{*}$ values of the EWMA- $\tilde{X}$ control chart in the presence of measurement error for different values of $\eta, \delta, n, B=1, m=1$

\begin{tabular}{ccccccc}
\hline$\delta$ & $\eta=0$ & $\eta=0.1$ & $\eta=0.2$ & $\eta=0.3$ & $\eta=0.5$ & $\eta=1$ \\
\hline 0.1 & $(0.1,0.3)$ & $(0.1,0.3)$ & $(0.1,0.3)$ & $(0.1,0.3)$ & $(0.1,0.3)$ & $(0.1,0.3)$ \\
0.2 & $(0.1,0.3)$ & $(0.1,0.3)$ & $(0.1,0.3)$ & $(0.1,0.3)$ & $(0.1,0.3)$ & $(0.1,0.3)$ \\
0.3 & $(0.1,0.3)$ & $(0.1,0.3)$ & $(0.1,0.3)$ & $(0.1,0.3)$ & $(0.1,0.3)$ & $(0.1,0.3)$ \\
0.5 & $(0.1,0.4)$ & $(0.1,0.4)$ & $(0.1,0.4)$ & $(0.1,0.4)$ & $(0.1,0.4)$ & $(0.1,0.3)$ \\
0.7 & $(0.1,0.5)$ & $(0.1,0.5)$ & $(0.1,0.5)$ & $(0.1,0.5)$ & $(0.1,0.5)$ & $(0.1,0.4)$ \\
1.0 & $(0.2,0.7)$ & $(0.2,0.7)$ & $(0.2,0.7)$ & $(0.2,0.7)$ & $(0.2,0.7)$ & $(0.2,0.5)$ \\
1.5 & $(0.4,1.1)$ & $(0.4,1.1)$ & $(0.4,1.0)$ & $(0.4,1.0)$ & $(0.4,1.0)$ & $(0.3,0.8)$ \\
2.0 & $(0.7,1.5)$ & $(0.7,1.4)$ & $(0.7,1.4)$ & $(0.6,1.4)$ & $(0.6,1.3)$ & $(0.4,1.0)$ \\
\hline
\end{tabular}




\begin{tabular}{ccccccc}
\hline$\delta$ & $\eta=0$ & $\eta=0.1$ & $\eta=0.2$ & $\eta=0.3$ & $\eta=0.5$ & $\eta=1$ \\
\hline 0.1 & $(0.1,0.2)$ & $(0.1,0.2)$ & $(0.1,0.2)$ & $(0.1,0.2)$ & $(0.1,0.2)$ & $(0.1,0.2)$ \\
0.2 & $(0.1,0.2)$ & $(0.1,0.2)$ & $(0.1,0.2)$ & $(0.1,0.2)$ & $(0.1,0.2)$ & $(0.1,0.2)$ \\
0.3 & $(0.1,0.2)$ & $(0.1,0.2)$ & $(0.1,0.2)$ & $(0.1,0.2)$ & $(0.1,0.2)$ & $(0.1,0.2)$ \\
0.5 & $(0.1,0.4)$ & $(0.1,0.4)$ & $(0.1,0.4)$ & $(0.1,0.4)$ & $(0.1,0.3)$ & $(0.1,0.3)$ \\
0.7 & $(0.2,0.5)$ & $(0.2,0.5)$ & $(0.2,0.5)$ & $(0.2,0.5)$ & $(0.2,0.5)$ & $(0.1,0.4)$ \\
1.0 & $(0.3,0.7)$ & $(0.3,0.7)$ & $(0.3,0.7)$ & $(0.3,0.7)$ & $(0.3,0.6)$ & $(0.2,0.5)$ \\
1.5 & $(0.6,1.1)$ & $(0.6,1.1)$ & $(0.6,1.1)$ & $(0.6,1.0)$ & $(0.5,1.0)$ & $(0.4,0.8)$ \\
2.0 & $(0.9,1.4)$ & $(0.8,1.4)$ & $(0.8,1.4)$ & $(0.8,1.4)$ & $(0.8,1.3)$ & $(0.6,1.0)$ \\
\hline
\end{tabular}

\begin{tabular}{ccccccc}
\hline$\delta$ & $\eta=0$ & $\eta=0.1$ & $\eta=0.2$ & $\eta=0.3$ & $\eta=0.5$ & $\eta=1$ \\
\hline 0.1 & $(0.1,0.2)$ & $(0.1,0.2)$ & $(0.1,0.2)$ & $(0.1,0.2)$ & $(0.1,0.2)$ & $(0.1,0.2)$ \\
0.2 & $(0.1,0.2)$ & $(0.1,0.2)$ & $(0.1,0.2)$ & $(0.1,0.2)$ & $(0.1,0.2)$ & $(0.1,0.2)$ \\
0.3 & $(0.1,0.2)$ & $(0.1,0.2)$ & $(0.1,0.2)$ & $(0.1,0.2)$ & $(0.1,0.2)$ & $(0.1,0.2)$ \\
0.5 & $(0.2,0.4)$ & $(0.2,0.4)$ & $(0.2,0.4)$ & $(0.1,0.4)$ & $(0.1,0.3)$ & $(0.1,0.3)$ \\
0.7 & $(0.3,0.5)$ & $(0.3,0.5)$ & $(0.3,0.5)$ & $(0.2,0.5)$ & $(0.2,0.5)$ & $(0.2,0.4)$ \\
1.0 & $(0.4,0.7)$ & $(0.4,0.7)$ & $(0.4,0.7)$ & $(0.4,0.7)$ & $(0.4,0.6)$ & $(0.3,0.5)$ \\
1.5 & $(0.8,1.1)$ & $(0.8,1.1)$ & $(0.7,1.1)$ & $(0.7,1.0)$ & $(0.7,1.0)$ & $(0.5,0.8)$ \\
2.0 & $(0.9,1.3)$ & $(0.9,1.3)$ & $(0.9,1.3)$ & $(0.9,1.3)$ & $(0.9,1.2)$ & $(0.7,1.0)$ \\
\hline
\end{tabular}

\begin{tabular}{ccccccc}
\hline$\delta$ & $\eta=0$ & $\eta=0.1$ & $\eta=0.2$ & $\eta=0.3$ & $\eta=0.5$ & $\eta=1$ \\
\hline 0.1 & $(0.1,0.2)$ & $(0.1,0.2)$ & $(0.1,0.2)$ & $(0.1,0.2)$ & $(0.1,0.2)$ & $(0.1,0.2)$ \\
0.2 & $(0.1,0.2)$ & $(0.1,0.2)$ & $(0.1,0.2)$ & $(0.1,0.2)$ & $(0.1,0.2)$ & $(0.1,0.2)$ \\
0.3 & $(0.1,0.2)$ & $(0.1,0.2)$ & $(0.1,0.2)$ & $(0.1,0.2)$ & $(0.1,0.2)$ & $(0.1,0.2)$ \\
0.5 & $(0.2,0.4)$ & $(0.2,0.4)$ & $(0.2,0.4)$ & $(0.2,0.4)$ & $(0.2,0.3)$ & $(0.1,0.3)$ \\
0.7 & $(0.3,0.5)$ & $(0.3,0.5)$ & $(0.3,0.5)$ & $(0.3,0.5)$ & $(0.3,0.5)$ & $(0.2,0.4)$ \\
1.0 & $(0.5,0.7)$ & $(0.5,0.7)$ & $(0.5,0.7)$ & $(0.5,0.7)$ & $(0.4,0.6)$ & $(0.3,0.5)$ \\
1.5 & $(0.8,1.1)$ & $(0.8,1.1)$ & $(0.8,1.1)$ & $(0.8,1.1)$ & $(0.8,1.1)$ & $(0.6,0.8)$ \\
2.0 & $(1.0,1.2)$ & $(1.0,1.2)$ & $(1.0,1.2)$ & $(1.0,1.2)$ & $(0.9,1.2)$ & $(0.8,1.0)$ \\
\hline
\end{tabular}

Some simple conclusions can be drawn from this table as follows.

Given $n, \delta, B$ and $m=1$, when $\eta$ increases, the values of $\lambda^{*}$ and $K^{*}$ decrease. For instance, when $n=5, B=1, m=1$ and $\delta=1.5$, we have $\lambda^{*}=0.6$ and $K^{*}=1.1$ for $\eta=0$ (process is free of measurement error) and $\lambda^{*}=0.5$ and $K^{*}=1.0$ for $\eta=0.5$.

When $\delta \leq 0.3$, given $n$, B and $m=1$, values of $\lambda^{*}$ and $K^{*}$ are constants irrespective of the value of $\eta$. For instance, when $n=5, B=1, m=1$ and $\delta=0.2$, we have $\lambda^{*}=0.1$ and $K^{*}=0.2$ for $\eta=0$ (process is free of measurement error) and $\lambda^{*}$ $=0.1$ and $K^{*}=0.2$ for $\eta=1$.

Using the optimal couples $\left(\lambda^{*}, K^{*}\right)$ in Table 2, the values of $A R L$ and $S D R L$ can be calculated as in (18) and (19). The results corresponding to the same values of parameters in Table 2 are presented in Table 3. 
Table 3. $A R L$ and $S D R L$ values of the EWMA- $\tilde{X}$ control chart in the presence of measurement error for different values of $\eta, \delta, n, B=1, m=1$

\begin{tabular}{ccccccc}
\hline$\delta$ & $\eta=0$ & $\eta=0.1$ & $\eta=0.2$ & $\eta=0.3$ & $\eta=0.5$ & $\eta=1$ \\
\hline 0.1 & $(146.3,130.4)$ & $(147.1,131.2)$ & $(149.6,133.7)$ & $(153.7,137.8)$ & $(165.8,150.0)$ & $(208.2,192.7)$ \\
0.2 & $(56.4,42.0)$ & $(56.9,42.4)$ & $(58.1,43.6)$ & $(60.2,45.5)$ & $(66.6,51.7)$ & $(93.4,77.8)$ \\
0.3 & $(31.0,19.0)$ & $(31.2,19.2)$ & $(31.9,19.8)$ & $(33.0,20.7)$ & $(36.5,23.7)$ & $(51.7,37.6)$ \\
0.5 & $(14.9,8.1)$ & $(15.0,8.1)$ & $(15.3,8.3)$ & $(15.8,8.7)$ & $(17.5,9.7)$ & $(24.6,14.0)$ \\
0.7 & $(9.0,4.6)$ & $(9.1,4.7)$ & $(9.3,4.8)$ & $(9.6,5.0)$ & $(10.6,5.6)$ & $(15.1,8.2)$ \\
1.0 & $(5.2,2.5)$ & $(5.3,2.6)$ & $(5.4,2.6)$ & $(5.6,2.7)$ & $(6.2,3.1)$ & $(8.8,4.6)$ \\
1.5 & $(2.8,1.3)$ & $(2.9,1.3)$ & $(2.9,1.4)$ & $(3.0,1.4)$ & $(3.4,1.6)$ & $(4.8,2.3)$ \\
2.0 & $(1.8,0.9)$ & $(1.8,0.9)$ & $(1.9,0.9)$ & $(1.9,1.0)$ & $(2.2,1.0)$ & $(3.1,1.4)$ \\
\hline
\end{tabular}

\begin{tabular}{ccccccc}
\hline$\delta$ & $\eta=0$ & $\eta=0.1$ & $\eta=0.2$ & $\eta=0.3$ & $\eta=0.5$ & $\eta=1$ \\
\hline 0.1 & $(110.5,94.6)$ & $(111.2,95.4)$ & $(113.4,97.5)$ & $(116.9,101.1)$ & $(127.6,111.7)$ & $(167.8,152.0)$ \\
0.2 & $(40.5,27.3)$ & $(40.8,27.5)$ & $(41.7,28.3)$ & $(43.1,29.7)$ & $(47.8,33.9)$ & $(67.7,52.7)$ \\
0.3 & $(22.6,12.8)$ & $(22.7,12.9)$ & $(23.2,13.2)$ & $(24.0,13.7)$ & $(26.4,15.2)$ & $(37.1,24.3)$ \\
0.5 & $(10.6,5.6)$ & $(10.7,5.6)$ & $(11.0,5.8)$ & $(11.4,6.0)$ & $(12.6,6.7)$ & $(17.8,9.8)$ \\
0.7 & $(6.4,3.2)$ & $(6.5,3.2)$ & $(6.6,3.3)$ & $(6.8,3.4)$ & $(7.6,3.8)$ & $(10.8,5.7)$ \\
1.0 & $(3.7,1.7)$ & $(3.8,1.8)$ & $(3.8,1.8)$ & $(4.0,1.9)$ & $(4.4,2.1)$ & $(6.3,3.1)$ \\
1.5 & $(2.0,1.0)$ & $(2.0,1.0)$ & $(2.1,1.0)$ & $(2.1,1.0)$ & $(2.4,1.1)$ & $(3.4,1.6)$ \\
2.0 & $(1.3,0.6)$ & $(1.3,0.6)$ & $(1.3,0.6)$ & $(1.4,0.6)$ & $(1.5,0.7)$ & $(2.2,1.1)$ \\
\hline
\end{tabular}

\begin{tabular}{ccccccc}
\hline$\delta$ & $\eta=0$ & $\eta=0.1$ & $\eta=0.2$ & $\eta=0.3$ & $\eta=0.5$ & $\eta=1$ \\
\hline 0.1 & $(89.3,73.7)$ & $(89.9,74.4)$ & $(91.8,76.2)$ & $(94.9,79.2)$ & $(104.2,88.4)$ & $(140.8,124.9)$ \\
0.2 & $(32.2,20.1)$ & $(32.5,20.3)$ & $(33.2,20.9)$ & $(34.3,21.9)$ & $(38.0,25.0)$ & $(53.8,39.5)$ \\
0.3 & $(18.0,10.0)$ & $(18.2,10.1)$ & $(18.6,10.3)$ & $(19.2,10.7)$ & $(21.2,11.9)$ & $(29.6,17.8)$ \\
0.5 & $(8.4,4.3)$ & $(8.5,4.4)$ & $(8.7,4.5)$ & $(9.0,4.6)$ & $(10.0,5.2)$ & $(14.2,7.7)$ \\
0.7 & $(5.1,2.5)$ & $(5.1,2.5)$ & $(5.2,2.5)$ & $(5.4,2.6)$ & $(6.0,3.0)$ & $(8.6,4.4)$ \\
1.0 & $(3.0,1.4)$ & $(3.0,1.4)$ & $(3.0,1.4)$ & $(3.2,1.5)$ & $(3.5,1.6)$ & $(5.0,2.4)$ \\
1.5 & $(1.6,0.8)$ & $(1.6,0.8)$ & $(1.6,0.8)$ & $(1.7,0.8)$ & $(1.9,0.9)$ & $(2.7,1.3)$ \\
2.0 & $(1.1,0.3)$ & $(1.1,0.3)$ & $(1.1,0.3)$ & $(1.1,0.4)$ & $(1.2,0.5)$ & $(1.7,0.8)$ \\
\hline
\end{tabular}

\begin{tabular}{ccccccc}
\hline$\delta$ & $\eta=0$ & $\eta=0.1$ & $\eta=0.2$ & $\eta=0.3$ & $\eta=0.5$ & $\eta=1$ \\
\hline 0.1 & $(75.4,60.1)$ & $(75.9,60.7)$ & $(77.5,62.2)$ & $(80.2,64.8)$ & $(88.5,72.9)$ & $(121.6,105.7)$ \\
0.2 & $(27.2,15.9)$ & $(27.4,16.0)$ & $(28.0,16.5)$ & $(28.9,17.3)$ & $(31.9,19.8)$ & $(45.1,31.5)$ \\
0.3 & $(15.2,8.3)$ & $(15.3,8.3)$ & $(15.6,8.5)$ & $(16.2,8.9)$ & $(17.9,9.9)$ & $(25.0,14.3)$ \\
0.5 & $(7.0,3.5)$ & $(7.1,3.6)$ & $(7.3,3.7)$ & $(7.5,3.8)$ & $(8.3,4.3)$ & $(11.9,6.3)$ \\
0.7 & $(4.2,2.0)$ & $(4.3,2.0)$ & $(4.4,2.1)$ & $(4.5,2.2)$ & $(5.0,2.4)$ & $(7.2,3.6)$ \\
1.0 & $(2.5,1.2)$ & $(2.5,1.2)$ & $(2.5,1.2)$ & $(2.6,1.2)$ & $(2.9,1.4)$ & $(4.2,2.0)$ \\
1.5 & $(1.3,0.6)$ & $(1.3,0.6)$ & $(1.3,0.6)$ & $(1.4,0.6)$ & $(1.5,0.8)$ & $(2.2,1.1)$ \\
2.0 & $(1.0,0.2)$ & $(1.0,0.2)$ & $(1.0,0.2)$ & $(1.0,0.2)$ & $(1.1,0.3)$ & $(1.4,0.7)$ \\
\hline
\end{tabular}

The obtained results show that, given the value of $n, \delta, m=1$ and $B=1$, the smaller the precision error ratio $\eta$, the faster the control chart in the detection of the out-of-control condition, demonstrating the negative effect of the measurement error on the EWMA- $\tilde{X}$ 's performance. For instance, when $n=5, B=1, m=1$ and $\delta=0.2$, we have $A R L=$ 40.5 and $S D R L=27.3$ for $\eta=0$ (process is free of measurement error) and $A R L=41.7$ and $S D R L=28.3$ for $\eta=0.2$ (see Table 3). 
Table 4. $A R L$ and $S D R L$ values of the EWMA- $\tilde{X}$ control chart in the presence of measurement error for different values of $B, \delta, n, \eta=0.28, m=1$

\begin{tabular}{cccccc}
\hline$\delta$ & $B=1$ & $B=2$ & $B=3$ & $B=4$ & $B=5$ \\
\hline 0.1 & $(152.8,136.9)$ & $(147.9,132.0)$ & $(147.0,131.1)$ & $(146.7,130.8)$ & $(146.5,130.6)$ \\
0.2 & $(59.7,45.1)$ & $(57.3,42.8)$ & $(56.8,42.3)$ & $(56.6,42.2)$ & $(56.6,42.1)$ \\
0.3 & $(32.8,20.5)$ & $(31.5,19.4)$ & $(31.2,19.2)$ & $(31.1,19.1)$ & $(31.1,19.1)$ \\
0.5 & $(15.7,8.6)$ & $(15.1,8.2)$ & $(15.0,8.1)$ & $(14.9,8.1)$ & $(14.9,8.1)$ \\
0.7 & $(9.5,4.9)$ & $(9.1,4.7)$ & $(9.0,4.7)$ & $(9.0,4.7)$ & $(9.0,4.6)$ \\
1.0 & $(5.5,2.7)$ & $(5.3,2.6)$ & $(5.3,2.6)$ & $(5.3,2.6)$ & $(5.2,2.5)$ \\
1.5 & $(3.0,1.4)$ & $(2.9,1.3)$ & $(2.9,1.3)$ & $(2.9,1.3)$ & $(2.8,1.3)$ \\
2.0 & $(1.9,0.9)$ & $(1.8,0.9)$ & $(1.8,0.9)$ & $(1.8,0.9)$ & $(1.8,0.9)$ \\
\hline
\end{tabular}

\begin{tabular}{cccccc}
\hline$\delta$ & $B=1$ & $B=2$ & $B=3$ & $B=4$ & $B=5$ \\
\hline 0.1 & $(116.1,100.2)$ & $(111.9,96.1)$ & $(111.1,95.3)$ & $(110.8,95.0)$ & $(110.7,94.9)$ \\
0.2 & $(42.8,29.4)$ & $(41.0,27.8)$ & $(40.7,27.5)$ & $(40.6,27.4)$ & $(40.6,27.4)$ \\
0.3 & $(23.8,13.6)$ & $(22.9,13.0)$ & $(22.7,12.9)$ & $(22.6,12.8)$ & $(22.6,12.8)$ \\
0.5 & $(11.3,5.9)$ & $(10.8,5.7)$ & $(10.7,5.6)$ & $(10.7,5.6)$ & $(10.7,5.6)$ \\
0.7 & $(6.8,3.4)$ & $(6.5,3.2)$ & $(6.4,3.2)$ & $(6.4,3.2)$ & $(6.4,3.2)$ \\
1.0 & $(3.9,1.9)$ & $(3.8,1.8)$ & $(3.8,1.8)$ & $(3.7,1.8)$ & $(3.7,1.8)$ \\
1.5 & $(2.1,1.0)$ & $(2.0,1.0)$ & $(2.0,1.0)$ & $(2.0,1.0)$ & $(2.0,1.0)$ \\
2.0 & $(1.4,0.6)$ & $(1.3,0.6)$ & $(1.3,0.6)$ & $(1.3,0.6)$ & $(1.3,0.6)$ \\
\hline
\end{tabular}

\begin{tabular}{cccccc}
\hline$\delta$ & $B=1$ & $B=2$ & $B=2$ & $B=4$ & $B=5$ \\
\hline 0.1 & $(94.2,78.5)$ & $(90.5,74.9)$ & $(89.9,74.3)$ & $(89.6,74.0)$ & $(89.5,73.9)$ \\
0.2 & $(34.1,21.6)$ & $(32.7,20.5)$ & $(32.5,20.2)$ & $(32.4,20.2)$ & $(32.3,20.1)$ \\
0.3 & $(19.0,10.6)$ & $(18.3,10.2)$ & $(18.1,10.1)$ & $(18.1,10.0)$ & $(18.1,10.0)$ \\
0.5 & $(8.9,4.6)$ & $(8.6,4.4)$ & $(8.5,4.4)$ & $(8.5,4.3)$ & $(8.4,4.3)$ \\
0.7 & $(5.4,2.6)$ & $(5.1,2.5)$ & $(5.1,2.5)$ & $(5.1,2.5)$ & $(5.1,2.5)$ \\
1.0 & $(3.1,1.4)$ & $(3.0,1.4)$ & $(3.0,1.4)$ & $(3.0,1.4)$ & $(3.0,1.4)$ \\
1.5 & $(1.7,0.8)$ & $(1.6,0.8)$ & $(1.6,0.8)$ & $(1.6,0.8)$ & $(1.6,0.8)$ \\
2.0 & $(1.1,0.4)$ & $(1.1,0.3)$ & $(1.1,0.3)$ & $(1.1,0.3)$ & $(1.1,0.3)$ \\
\hline
\end{tabular}

\begin{tabular}{cccccc}
\hline$\delta$ & $B=1$ & $B=2$ & $B=3$ & $B=4$ & $B=5$ \\
\hline 0.1 & $(79.6,64.2)$ & $(76.4,61.2)$ & $(75.8,60.6)$ & $(75.6,60.4)$ & $(75.5,60.3)$ \\
0.2 & $(28.7,17.1)$ & $(27.6,16.2)$ & $(27.4,16.0)$ & $(27.3,15.9)$ & $(27.3,15.9)$ \\
0.3 & $(16.0,8.8)$ & $(15.4,8.4)$ & $(15.3,8.3)$ & $(15.2,8.3)$ & $(15.2,8.3)$ \\
0.5 & $(7.5,3.8)$ & $(7.1,3.6)$ & $(7.1,3.6)$ & $(7.1,3.6)$ & $(7.1,3.6)$ \\
0.7 & $(4.5,2.1)$ & $(4.3,2.0)$ & $(4.3,2.0)$ & $(4.2,2.0)$ & $(4.2,2.0)$ \\
1.0 & $(2.6,1.2)$ & $(2.5,1.2)$ & $(2.5,1.2)$ & $(2.5,1.2)$ & $(2.5,1.2)$ \\
1.5 & $(1.4,0.6)$ & $(1.3,0.6)$ & $(1.3,0.6)$ & $(1.3,0.6)$ & $(1.3,0.6)$ \\
2.0 & $(1.0,0.2)$ & $(1.0,0.2)$ & $(1.0,0.2)$ & $(1.0,0.2)$ & $(1.0,0.2)$ \\
\hline
\end{tabular}

Table 4 presents the performance of the EWMA- $\tilde{X}$ chart with linear covariate error model under the effect of $B \in\{1,2$, $3,4,5\}$ for $\eta=0.28$ and $m=1$ and different combinations of $\delta \in\{0,0.1,0.2,0.3,0.5,0.7,1.0,1.5,2.0\}$ and $n \in\{3,5,7,9\}$. As can be seen from this table, when B increases, given $n, \delta, \eta$ and $m$, the negative effect of the measurement error on the performance of the EWMA- $\tilde{X}$ chart decreases. For instance, when $n=5, \eta=0.28, m=1$ and $\delta=0.2$, we have $A R L=42.8$ 
and $S D R L=29.4$ for $B=1$ and $A R L=40.6$ and $S D R L=27.4$ for $B=4$ (see Table 4).

In order to compensate for the effect of measurement error, Linna and Woodall ${ }^{[18]}$ recommended to take multiple measurements per item in each sample.

Table 5. $A R L$ and SDRL values of the EWMA- $\tilde{X}$ control chart in the presence of measurement error for different values of $m, \delta, n, \eta=0.28, B=1$

\begin{tabular}{cccccc}
\hline$\delta$ & $m=1$ & $m=3$ & $m=5$ & $m=7$ & $m=10$ \\
\hline 0.1 & $(152.8,136.9)$ & $(148.5,132.6)$ & $(147.6,131.7)$ & $(147.2,131.3)$ & $(146.9,131.0)$ \\
0.2 & $(59.7,45.1)$ & $(57.5,43.0)$ & $(57.1,42.6)$ & $(56.9,42.4)$ & $(56.8,42.3)$ \\
0.3 & $(32.8,20.5)$ & $(31.6,19.5)$ & $(31.4,19.3)$ & $(31.3,19.2)$ & $(31.2,19.2)$ \\
0.5 & $(15.7,8.6)$ & $(15.1,8.2)$ & $(15.0,8.2)$ & $(15.0,8.1)$ & $(14.9,8.1)$ \\
0.7 & $(9.5,4.9)$ & $(9.2,4.7)$ & $(9.1,4.7)$ & $(9.1,4.7)$ & $(9.0,4.7)$ \\
1.0 & $(5.5,2.7)$ & $(5.3,2.6)$ & $(5.3,2.6)$ & $(5.3,2.6)$ & $(5.3,2.6)$ \\
1.5 & $(3.0,1.4)$ & $(2.9,1.3)$ & $(2.9,1.3)$ & $(2.9,1.3)$ & $(2.9,1.3)$ \\
2.0 & $(1.9,0.9)$ & $(1.9,0.9)$ & $(1.8,0.9)$ & $(1.8,0.9)$ & $(1.8,0.9)$ \\
\hline
\end{tabular}

$n=5$

\begin{tabular}{cccccc}
\hline$\delta$ & $m=1$ & $m=3$ & $m=5$ & $m=7$ & $m=10$ \\
\hline 0.1 & $(116.1,100.2)$ & $(112.4,96.5)$ & $(111.6,95.8)$ & $(111.3,95.5)$ & $(111.1,95.2)$ \\
0.2 & $(42.8,29.4)$ & $(41.2,28.0)$ & $(40.9,27.7)$ & $(40.8,27.6)$ & $(40.7,27.5)$ \\
0.3 & $(23.8,13.6)$ & $(23.0,13.0)$ & $(22.8,12.9)$ & $(22.7,12.9)$ & $(22.7,12.9)$ \\
0.5 & $(11.3,5.9)$ & $(10.9,5.7)$ & $(10.8,5.7)$ & $(10.7,5.6)$ & $(10.7,5.6)$ \\
0.7 & $(6.8,3.4)$ & $(6.5,3.3)$ & $(6.5,3.2)$ & $(6.5,3.2)$ & $(6.4,3.2)$ \\
1.0 & $(3.9,1.9)$ & $(3.8,1.8)$ & $(3.8,1.8)$ & $(3.8,1.8)$ & $(3.8,1.8)$ \\
1.5 & $(2.1,1.0)$ & $(2.1,1.0)$ & $(2.1,1.0)$ & $(2.1,1.0)$ & $(2.1,1.0)$ \\
2.0 & $(1.4,0.6)$ & $(1.3,0.6)$ & $(1.3,0.6)$ & $(1.3,0.6)$ & $(1.3,0.6)$ \\
\hline
\end{tabular}

\begin{tabular}{cccccc}
\hline$\delta$ & $m=1$ & $m=3$ & $m=5$ & $m=7$ & $m=10$ \\
\hline 0.1 & $(94.2,78.5)$ & $(91.0,75.3)$ & $(90.3,74.7)$ & $(90.0,74.4)$ & $(89.8,74.2)$ \\
0.2 & $(34.1,21.6)$ & $(32.9,20.6)$ & $(32.6,20.4)$ & $(32.5,20.3)$ & $(32.4,20.2)$ \\
0.3 & $(19.0,10.6)$ & $(18.4,10.2)$ & $(18.2,10.1)$ & $(18.2,10.1)$ & $(18.1,10.1)$ \\
0.5 & $(8.9,4.6)$ & $(8.6,4.4)$ & $(8.5,4.4)$ & $(8.5,4.4)$ & $(8.5,4.3)$ \\
0.7 & $(5.4,2.6)$ & $(5.2,2.5)$ & $(5.1,2.5)$ & $(5.1,2.5)$ & $(5.1,2.5)$ \\
1.0 & $(3.1,1.4)$ & $(3.0,1.4)$ & $(3.0,1.4)$ & $(3.0,1.4)$ & $(3.0,1.4)$ \\
1.5 & $(1.7,0.8)$ & $(1.6,0.8)$ & $(1.6,0.8)$ & $(1.6,0.8)$ & $(1.6,0.8)$ \\
2.0 & $(1.1,0.4)$ & $(1.1,0.3)$ & $(1.1,0.3)$ & $(1.1,0.3)$ & $(1.1,0.3)$ \\
\hline
\end{tabular}

$n=9$

\begin{tabular}{cccccc}
\hline$\delta$ & $m=1$ & $m=3$ & $m=5$ & $m=7$ & $m=10$ \\
\hline 0.1 & $(79.6,64.2)$ & $(76.8,61.5)$ & $(76.2,61.0)$ & $(76.0,60.7)$ & $(75.8,60.5)$ \\
0.2 & $(28.7,17.1)$ & $(27.7,16.3)$ & $(27.5,16.1)$ & $(27.4,16.0)$ & $(27.4,16.0)$ \\
0.3 & $(16.0,8.8)$ & $(15.4,8.4)$ & $(15.3,8.4)$ & $(15.3,8.3)$ & $(15.2,8.3)$ \\
0.5 & $(7.5,3.8)$ & $(7.2,3.6)$ & $(7.1,3.6)$ & $(7.1,3.6)$ & $(7.1,3.6)$ \\
0.7 & $(4.5,2.1)$ & $(4.3,2.1)$ & $(4.3,2.0)$ & $(4.3,2.0)$ & $(4.3,2.0)$ \\
1.0 & $(2.6,1.2)$ & $(2.5,1.2)$ & $(2.5,1.2)$ & $(2.5,1.2)$ & $(2.5,1.2)$ \\
1.5 & $(1.4,0.6)$ & $(1.3,0.6)$ & $(1.3,0.6)$ & $(1.3,0.6)$ & $(1.3,0.6)$ \\
2.0 & $(1.0,0.2)$ & $(1.0,0.2)$ & $(1.0,0.2)$ & $(1.0,0.2)$ & $(1.0,0.2)$ \\
\hline
\end{tabular}


In this study, we suppose that the number $m$ of multiple measurement per item takes the values in $\{1,3,5,7,10\}$. Table 5 demonstrates the effect of $\mathrm{m}$ on the values of $A R L$ for the case $\eta=0.28, B=1, n \in\{3,5,7,9\}$ and $\delta \in\{0,0.1,0.2$, $0.3,0.5,0.7,1.0,1.5,2.0\}$. The obtained results show that when $\mathrm{m}$ increases, both $A R L$ and $S D R L$ decrease, demonstrating the positive effect of the number of repeated measurements $m$ per item on the EWMA- $\tilde{X}$ chart's performance. For instance, when $n=5, \eta=0.28$ and $\delta=0.2$, we have $A R L=42.8$ and $S D R L=29.4$ for $m=1$ and $A R L=40.9$ and $S D R L=$ 27.7 for $m=5$ (see Table 5).

In practice, the quality practitioners often have the interest to detect a range of shifts $\delta \in \Omega=[\mathrm{a}, \mathrm{b}]$, but no preference for any particular size of the process shift, the use of the uniform distribution has been proposed to account for the unknown shift size in several papers (for instance, see ${ }^{[9,8]}$ ) and the statistical performance of the corresponding chart can be evaluated through the EARL (Expected Average Run Length) defined as

$$
E A R L=\int_{\Omega} A R L \times f_{\delta}(\delta) d \delta
$$

with $f_{\delta}(\delta)=\frac{1}{b-a}$ for $\delta \in \Omega=[\mathrm{a}, \mathrm{b}]$ and $A R L$ is defined as in (18). In the following section, we will consider the specific range of shifts $\Omega=[0.1,2]$.

The values of $E A R L$ of the EWMA- $\tilde{X}$ chart with measurement error are plotted in Figure 1, for different combinations of measure precision error ratio $\eta \in[0,1], \Omega=[0.1,2], n \in\{3,5,7,9\}, m \in\{1,5\}$ and $B=1$.

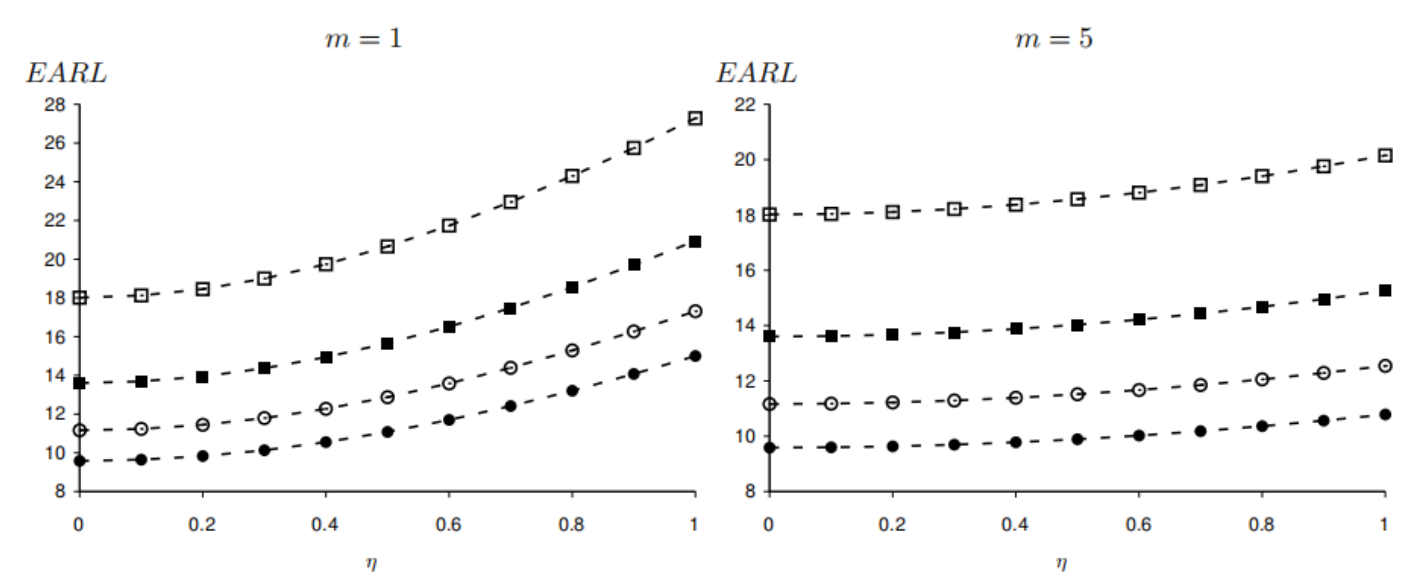

Figure 1. The effect of $\boldsymbol{\eta}$ on the overall performance of the EWMA- $\tilde{X}$ control chart in the presence of measurement error for $\mathbf{r} n=3(-\square-)$, $n=5(-\square-), n=7(-\odot), n=9(-\bullet-), A R L_{0}=370.4, B=1, m \in\{1,5\},, \Omega=[0.1,2]$

The EARL values in Figure 1 show a similar tendency as for the deterministic shift size results discussed above. When the precision error ratio $\eta$ increases, the overall performance of the EWMA- $\tilde{X}$ chart deteriorates. For instance, when $n=$ $3, B=1$ and $m=1$, we have $E A R L=18.02$ for $\eta=0$ (process is free of measurement error) and $E A R L=18.46$ for $\eta=0.2$ (see Figure 1). Furthermore, with $m=5$, the values of $E A R L$ in presence of measurement error are approximately the same as the values of EARL without measurement error(i.e. $\eta=0$ ) when $\eta \leq 0.28$. For example, when $n=3, B=1$ and $m=5$, we have $E A R L=18.02$ for $\eta=0$ (process is free of measurement error) and $E A R L=18.10$ for $\eta=0.2$ (see Figure 1). We can conclude that the precision error does not significantly affect the overall performance of the EWMA- $\tilde{X}$ control chart for the usual levels of accuracy error provided by calibrated gauges for the case of $m=5$ measurements per item.

Figure 2 displays the effect of B on the values of EARL of the EWMA- $\tilde{X}$ chart with measurement error for different combinations of $B \in[1,5], \Omega=[0.1,2], n \in\{3,5,7,9\}, m \in\{1,5\}$ and $\eta=0.28$. Similar to the case of deterministic shift size, an increase of the coefficient $B$ in the linear covariate model can reduce the negative effect of measurement errors on the overall performance of EWMA- $\tilde{X}$ chart. For instance, when $n=3, \eta=0.28, m=1$, we have $E A R L=18.87$ for $B=1$ and $E A R L=18.07$ for $B=4$ (see Figure 3). 

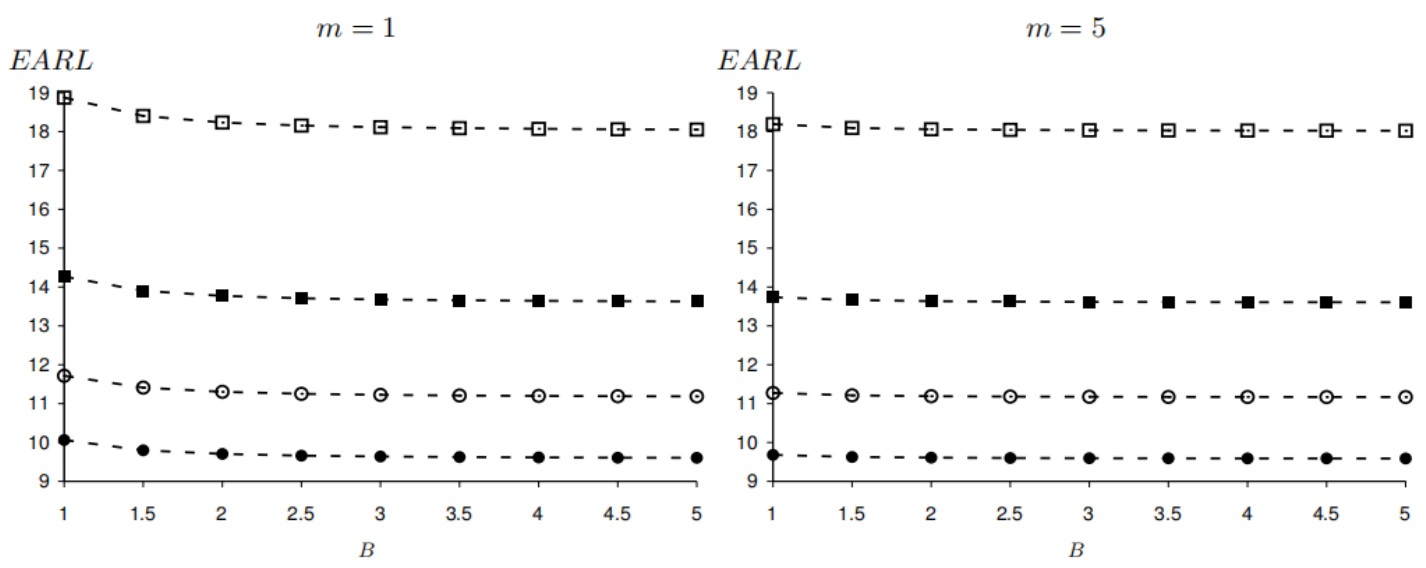

Figure 2. The effect of B on the overall performance of the EWMA- $\tilde{X}$ control chart in the presence of measurement error for $n=3(-\square-)$, $n=5(---), n=7(-\odot-), n=9(-\bullet-), A R L_{0}=370.4, B=1, m \in\{1,5\},, \Omega=[0.1,2]$

Finally, the effect of multiple measurements on the EWMA- $\tilde{X}$ chart performance is presented in Figure 3 for different combinations of $m \in\{1,3,5,7,10\}, n \in\{1,3,5,7\}, B=1, \Omega=[0.1,2]$ and $\eta=0.28$. When $m$ increases, the values of EARL decrease slightly. For example, when $n=3, \eta=0.28$, we have $E A R L=18.31$ for $m=3$ and $E A R L=18.10$ for $m$ $=10$ (see Figure 3 ). These findings in Figure 3 allow to reaffirm that, taking multiple measurements per item is a good strategy to compensate for the effect of measurement error.

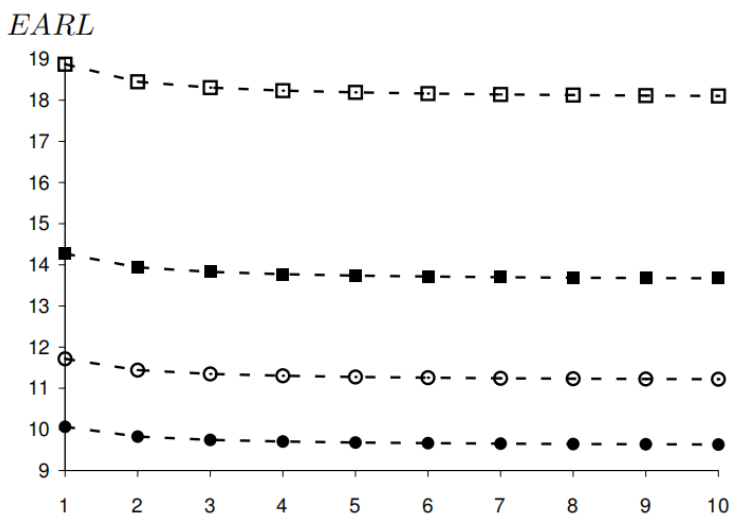

Figure 3. The effect of $m$ on the overall performance of the EWMA- $\tilde{X}$ control chart in the presence of measurement error for $n=3(-\square-)$, $n=5(-\square-), n=7(-\odot), n=9(-\bullet-), A R L_{0}=370.4, \eta=0.28, \eta=0.28, \Omega=[0.1,2]$

\section{Illustrative example}

To illustrate the use of the EWMA- $\tilde{X}$ chart in the presence of measurement error, let us consider a production process of $500 \mathrm{ml} \mathrm{milk} \mathrm{bottles} \mathrm{where} \mathrm{the} \mathrm{quality} \mathrm{characteristic} \mathrm{X}$ of interest is the weight (in ml) of each bottle. The context of the example presented here is similar to the one introduced in [6]. We assume that from a Phase I dataset, the following quantities have been estimated: $\mu_{0}=500.023$ and $\sigma_{0}=0.9616$. According to the quality practitioner in charge of this process, a shift of $0.5 \sigma_{0}$ (i.e. $\delta=0.5$ ) in the mean should be interpreted as a signal that something is going wrong in the production. For each subgroup, the sample size has been chosen to be $n=5$. Concerning the parameters of the twocomponent two-component measure-ment error, we assume $\eta=0.28, B=1, m=1$. We have the optimal chart parameters for the EWMA- $\tilde{X}$ chart are $K=0.1197$ and $\lambda=0.3716$.

Based on (10), the control limits of the EWMA- $\tilde{X}$ control chart with measurement error are equal to:

$$
\begin{aligned}
& L C L=500.023-0.1197 \times \sqrt{0.9616^{2}+(0.9616 \times 0.28)^{2}}=499.6649, \\
& L C L=500.023+0.1197 \times \sqrt{0.9616^{2}+(0.9616 \times 0.28)^{2}}=500.3811 .
\end{aligned}
$$


A Phase II dataset presented in Table 6. The first 10 subgroups are supposed to be in-control while the last 10 subgroups are supposed to have a lower milk weight, and thus, to be out-of-control. The corresponding sample median values $\tilde{\boldsymbol{X}}_{i}^{*}$ and the EWMA sequence $Z_{i}^{*}$ are presented in the two last columns of Table 6 and plotted in Figure 4, respectively. This figure confirms that from sample \#13 onward, the process is out-of-control.

Table 6. Illustrative example Phase II datasetx

\begin{tabular}{cccccccc}
\hline & & & $\boldsymbol{X}_{i, j}^{*}$ & & & $\tilde{\boldsymbol{X}}_{i}^{*}$ & $Z_{i}^{*}$ \\
\hline 1 & 500.46 & 498.99 & 500.22 & 500.41 & 498.96 & 500.22 & 500.047 \\
2 & 500.06 & 500.20 & 499.31 & 501.07 & 499.57 & 500.06 & 500.048 \\
3 & 498.82 & 501.55 & 499.48 & 499.20 & 501.56 & 499.48 & 499.980 \\
4 & 502.64 & 502.86 & 500.06 & 499.08 & 500.72 & 500.72 & 500.069 \\
5 & 500.06 & 500.03 & 500.09 & 498.88 & 497.64 & 500.03 & 500.064 \\
6 & 500.50 & 499.54 & 499.02 & 498.09 & 499.87 & 499.54 & 500.001 \\
7 & 498.89 & 500.20 & 501.10 & 502.01 & 500.99 & 500.99 & 500.120 \\
8 & 500.37 & 499.28 & 500.15 & 500.87 & 500.88 & 500.37 & 500.150 \\
9 & 499.81 & 500.62 & 500.68 & 500.67 & 500.00 & 500.62 & 500.206 \\
10 & 499.79 & 499.87 & 500.98 & 499.12 & 500.79 & 499.87 & 500.166 \\
11 & 500.28 & 500.47 & 500.26 & 498.60 & 500.65 & 500.28 & 500.179 \\
12 & 501.00 & 500.38 & 500.06 & 500.81 & 502.22 & 500.81 & 500.255 \\
13 & 499.92 & 500.13 & 501.46 & 502.29 & 502.78 & 501.46 & 500.399 \\
14 & 501.22 & 499.22 & 500.68 & 499.81 & 502.41 & 500.68 & 500.490 \\
15 & 500.68 & 501.93 & 499.55 & 502.51 & 500.91 & 500.91 & 500.490 \\
16 & 500.45 & 502.10 & 502.11 & 499.35 & 497.52 & 500.45 & 500.485 \\
17 & 500.51 & 498.56 & 498.87 & 501.05 & 500.52 & 500.51 & 500.488 \\
18 & 500.94 & 500.20 & 500.80 & 501.36 & 499.23 & 500.80 & 500.525 \\
19 & 500.15 & 500.29 & 500.83 & 499.91 & 498.93 & 500.15 & 500.480 \\
20 & 501.14 & 500.51 & 499.92 & 499.28 & 499.52 & 499.92 & 500.413 \\
\hline
\end{tabular}

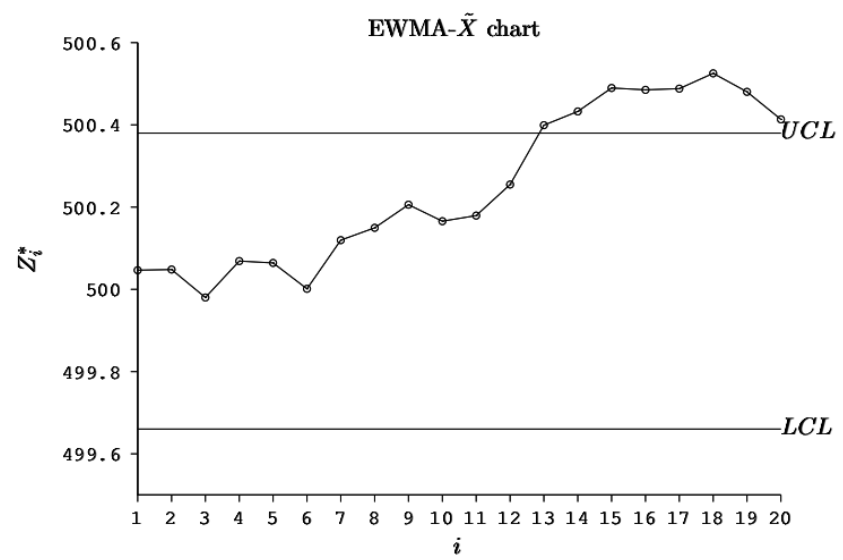

Figure 4. EWMA- $\tilde{X}$ control chart in the presence of measurement errorcorresponding to the Phase II data set in Table 6.

\section{Conclusions}

In this paper, we have corrected the distribution of the sample median used in [10] and then studied the effect of measurement error on the performance of the EWMA- $\tilde{X}$ control chart using a linear covariate error model. We have evaluated the overall performance of the EWMA- $\tilde{X}$ control using the $A R L, S D L R$ and $E A R L$ as performance metrics by using the Markov Chain-based approach proposed in [3]. Numerical results show that measurement error greatly affect the EWMA- $\tilde{X}$ chart's performance compared to the no measurement error case. With the precision error ratio increasing, the values of $\lambda^{*}$ and $K^{*}$ decrease and the performance of the EWMA- $\tilde{X}$ chart deteriorates. As a result, increasing the coefficient $\mathrm{B}$ in the linear covariate error model can reduce the negative effect of measurement error on the EWMA- $\tilde{X}$ chart. In order to compensate for the effect of measurement error on the EWMA- $\tilde{X}$ chart's performance, measuring each item several times is necessary, but increasing at the same time the cost of control. Future research about $\tilde{X}$ control charts should be focused on the extension to Run Rules type charts, on studying the effect of the parameters estimation on the Run Rules $\tilde{X}$ type charts statistical properties. 


\section{References}

[1] S. A. Abbasi. On the performance of ewma chart in the presence of two-component measurement error. Quality Engineering. 2010; 22(3): 199-213.

[2] Barry C Arnold, Narayanaswamy Balakrishnan, Haikady Navada Nagaraja. A first course in order statistics. 1992.

[3] D. Brook, D.A. Evans. An approach to the probability distribution of CUSUM run length. Biometrika. 1972; 59(3): 539-549.

[4] P. Castagliola. An $\bar{X}$ / R-EWMA control chart for monitoring the process sample median. International Journal of Reliability, Quality and Safety Engineering. 2001; 8(2): 123-135.

[5] P. Castagliola, F.O. Figueiredo. The median chart with estimated parameters. European Journal of Industrial Engineering. 2013; 7(5): 594-614.

[6] P. Castagliola, P. E. Maravelakis, F. O. Figueiredo. The EWMA Median chart with estimated parameters. IIE Transactions. 2016; 48(1): 66-74.

[7] Philippe Castagliola. Approximation of the normal sample median distribution using symmetrical johnson su distributions: application to quality control. Communications in Statistics-Simulation and Computation. 1998; 27(2): 289-301.

[8] G. Celano, P. Castagliola, G. Nenes, et al. Performance of t control charts in short runs with unknown shift sizes. Computers \& Industrial Engineering. 2013; 64: 56-68.

[9] A. Chen, Y. K. Chen. Design of EWMA and CUSUM control charts subject to random shift sizes and quality impacts. IIE Transactions. 2007; 39(12): 1127-1141.

[10] XiaoBin Cheng, FuKwun Wang. The performance of ewma median and cusum median control charts for a normal process with measurement errors. Quality and Reliability Engineering International. 2018; 34(2): 203-213.

[11] XiaoBin Cheng, FuKwun Wang. Vssi median control chart with estimated parameters and measurement errors. Quality and Reliability Engineering International. 2018; 34(5): 867-881.

[12] John T Chu. On the distribution of the sample median. The Annals of Mathematical Statistics. 1955; 112-116.

[13] A.F.B. Costa, P. Castagliola. Effect of Measurement Error and Autocorrelation on the $\bar{X}$ Chart. Journal of Applied Statistics. 2011; 38(4): 661-673.

14] Huu Du Nguyen, Quoc Thong Nguyen, Kim Phuc Tran, et al. On the performance of vsi shewhart control chart for monitoring the coefficient of variation in the presence of measurement errors. The International Journal of Advanced Manufacturing Technology. 2019; 104(1-4): 211-243.

[15] X.L. Hu, P. Castagliola, J. Sun, et al. The effect of measurement errors on the synthetic $\bar{X}$ chart. Quality and Reliability Engineering International. 2014.

[16] M.B.C. Khoo. A control chart based on sample median for the detection of a permanent shift in the process mean. Quality Engineering. 2005; 17(2): 243-257.

[17] G. Latouche, V. Ramaswami. Series on Statistics and Applied Probability. Introduction to Matrix Analytic Methods in Stochastic Modelling.SIAM, Philadelphia, PA; 1999.

[18] Kenneth W Linna, William H Woodall. Effect of measurement error on shewhart control charts. Journal of Quality technology. 2001; 33(2): 213-222.

[19] K.W. Linna, W.H. Woodall, K.L. Busby. The performance of multivariate control charts in the presence of measurement error. Journal of Quality Technology. 2001; 33(3): 349.

[20] P.E. Maravelakis. Measurement error effect on the CUSUM control chart. Journal of Applied Statistics. 2012; 39(2): $323-336$

[21] M.F. Neuts. Matrix-Geometric Solutions in Stochastic Models: an Algorithmic Approach. Johns Hopkins University Press, Baltimore, MD; 1981.

[22] R. Noorossana and Y. Zerehsaz. Effect of measurement error on phase II monitoring of simple linear profiles. The International Journal of Advanced Manufacturing Technology. 2015; 79(9-12): 2031-2040.

[23] S.H. Sheu, L. Yang. The generally weighted moving average control chart for monitoring the process median. Quality Engineering. 2006; 18(3): 333-344.

[24] Anan Tang, Philippe Castagliola, Xuelong Hu, et al. The performance of the adaptive ewma median chart in the presence of measurement error. Quality and Reliability Engineering International. 2019; 35(1): 423-438.

[25] Kim Phuc Tran, Philippe Castagliola, Narayanaswamy Balakrishnan. On the performance of shewhart median chart in the presence of measurement errors. Quality and Reliability Engineering International. 2017; 33(5): 1019-1029.

[26] Kim Phuc Tran, HD Nguyen, Quoc Thong Nguyen, et al. One-sided synthetic control charts for monitoring the coefficient of variation with measurement errors. International conference on industrial engineering and engineering management (IEEM). IEEE; 2018. p.1667-1671.

[27] Kim Phuc Tran, Huu Du Nguyen, Phuong Hanh Tran, et al. On the performance of cusum control charts 
for monitoring the coefficient of variation with measurement errors. The International Journal of Advanced Manufacturing Technology. 2019; 1-15.

[28] Phuong Hanh Tran, Kim Phuc Tran, Truong Thu Huong, et al. Real time data-driven approaches for credit card fraud detection. Proceedings of the 2018 International Conference on E-Business and Applications. 2018; 6-9.

[29] Phuong Hanh Tran, Kim Phuc Tran, Athanasios Rakitzis. A synthetic median control chart for monitoring the process mean with measurement errors. Quality and Reliability Engineering International. 2019; 35(4): 1100-1116.

[30] V. Vuong Trinh, K. Phuc Tran, T. Thu Huong. Data driven hyperparameter optimization of one - class support vector machines for anomaly detection in wireless sensor networks. International Conference on Advanced Technologies for Communications (ATC). 2017; 6-10. 